and Borg's proof of (7) for the case (8). In fact, even the use of (4) is ${ }^{6}$ between the lines of that step in Borg's proof of (7) in Liapounoff's case which leads from $[a, c]$ and $[c, b]$ to $[a, b]$, where $c$ is defined by $x(c)=\max x(t)$.

\title{
MAXIMUM SPEED IN STEADY SUBSONIC FLOWS*
}

\section{BY CHIA-SHUN YIH (University of Michigan)}

The purpose of this note is to show that the maximum speed in a singularity-free region of any steady subsonic flow must occur on its boundary, provided the flow is isentropic and irrotational. The corresponding result for irrotational flows of an incompressible fluid is well known.

Since the flow under consideration is irrotational, the square of the speed $q$ is given by

$$
q^{2}=\varphi_{. j} \varphi_{. j}
$$

in which $\varphi$ is the velocity potential, the summation convention is used, and, with Cartesian coordinates,

$$
\varphi_{. i}=\frac{\partial \varphi}{\partial x_{j}}
$$

is the $j$ th component of the velocity vector-with the usual minus sign suppressed for convenience. The equation of continuity is, for steady flow,

$$
\left(\rho \varphi_{, j}\right)_{, j}=0
$$

in which $\rho$ is the density. The equations of motion are

$$
(\ln \rho)_{, i}=-\frac{1}{2 c^{2}}\left(q^{2}+2 g x_{3}\right)_{, i}
$$

if $x_{3}$ is measured in a direction opposite to that of the gravitational acceleration $g$, and $c$ is the local speed of sound. Finally, the Bernoulli equation can be written in the form (with $p$ indicating pressure)

$$
c^{2}=k \frac{p_{0}}{\rho_{0}}-\frac{k-1}{2}\left(q^{2}+2 g x_{3}\right)
$$

in which $k$ is the ratio of the specific heat at constant pressure to that at constant volume, and the subscripts zero indicate that the quantities involved are taken at some point of reference, where the gas is at rest and from which $x_{3}$ is measured.

For a proof, it is sufficient to show that the Laplacian of $q^{2}$ is non-negative. Whereas this is easily done for irrotational flows of an incompressible fluid, it cannot be readily established for the flows under discussion. Professors D. Gilbarg (through his publications) and C. Truesdell (by oral communication) have indicated to the writer that

${ }^{6}$ G. Borg, Amer. J. Math. 71, 68 (1949); see also P. Hartman and A. Wintner, ibid., p. 209.

*Received April 18, 1957. 
the non-negativeness of $\nabla^{2} q^{2}$ can be replaced by that of

$$
\left(b_{i i}\left(q^{2}\right)_{, i i}\right.
$$

if the matrix $b_{i j}$ is positive definite. It will be seen that their suggestion provides one of the key steps to the following proof.

Taking the Laplacian of Eq. (1), one has

$$
\left(q^{2}\right)_{, i i}=\left(\varphi_{, i} \varphi_{. i}\right)_{, i i}=2 \varphi_{, i i} \varphi_{, i j}+2 \varphi_{, j} \varphi_{. i i i} .
$$

But Eq. (2) can be written as

$$
\varphi_{, i i}+(\ln \rho)_{. i} \varphi_{, i}=0
$$

so that

$$
\varphi_{, i} \varphi_{, i i i}=-\left[(\ln \rho)_{, i} \varphi_{, i}\right]_{, i} \varphi_{, i}=-(\ln \rho)_{, i} \varphi_{, i} \varphi_{, i}-(\ln \rho)_{, i i} \varphi_{, i} \varphi_{, i}
$$

and Eq. (5) becomes

$$
\left(q^{2}\right)_{, i i}=2 \varphi_{, i j} \varphi_{, i i}-2(\ln \rho)_{, i} \varphi_{, i i} \varphi_{, i}-2(\ln \rho)_{, i i} \varphi_{, i} \varphi_{, i} .
$$

By virtue of Eqs. (3) and (4), one has

$$
-2(\ln \rho)_{, i i} \varphi_{, i} \varphi_{, i}=\frac{1}{c^{2}}\left(q^{2}\right)_{, i j} \varphi_{, i} \varphi_{, i}+\frac{k-1}{2 c^{4}}\left(q^{2}+2 g x_{3}\right)_{, i}\left(q^{2}+2 q x_{3}\right)_{, i} \varphi_{, i} \varphi_{, i} .
$$

If

$$
2 \varphi_{, i} \varphi_{, i i}=\left(q^{2}\right)_{, i} \neq 0
$$

then $q^{2}$ cannot be a maximum. Therefore, one can concentrate on the case

$$
2 \varphi_{, j} \varphi_{, i i}=\left(q^{2}\right)_{, i}=0 .
$$

In this case Eq. (6) becomes, after substitution from Eqs. (7) and (8),

$$
\left(q^{2}\right)_{, i i}-\frac{1}{c^{2}} \varphi_{, i} \varphi_{, i}\left(q^{2}\right)_{, i i}=2 \varphi_{, i i} \varphi_{, i i}+\frac{2 g^{2}(k-1)}{c^{4}} \delta_{i 3} \delta_{i 3} \varphi_{, i} \varphi_{, i}
$$

or

$$
b_{i i}\left(q^{2}\right)_{, i j}=2 \varphi_{, i i} \varphi_{, i i}+\frac{2 g^{2}(k-1)}{c^{4}} \delta_{i 3} \delta_{i 3} \varphi_{, i} \varphi_{, i}=\text { positive }
$$

in which

$$
b_{i j}=\delta_{i i}-\frac{1}{c^{2}} \varphi_{. i} \varphi_{. i}, \text { and } \delta_{i i}=\text { the Kronecker delta. }
$$

Now since the flow is subsonic,

$$
\frac{\varphi \cdot \alpha \varphi \cdot \alpha}{c^{2}} \leq 1
$$

Then obviously if

$$
b_{i i}^{\prime}=\frac{\varphi_{, \alpha} \varphi_{, \alpha}}{c^{2}} \delta_{i j}-\frac{1}{c^{2}} \varphi_{, i} \varphi_{. i}
$$


is positive definite, $b_{i j}$ must also be. The proof will therefore be complete if one can demonstrate that $b_{i j}^{\prime}$ is positive definite, i.e., the bilinear form $b_{i j}^{\prime} u_{i} u_{i}$ is positive definite$u_{1}, u_{2}$, and $u_{3}$ being any three real numbers. But

$$
b_{i j}^{\prime} u_{i} u_{i}=\frac{1}{c^{2}}\left[\left(u_{1} \varphi_{, 2}-u_{2} \varphi_{, 1}\right)^{2}+\left(u_{2} \varphi_{, 3}-u_{3} \varphi_{, 2}\right)^{2}+\left(u_{3} \varphi_{, 1}-u_{1} \varphi_{, 3}\right)^{2}\right] .
$$

Thus $b_{i}^{\prime}$ and therefore $b_{i i}$ are positive definite, and Eq. (9) shows that the maximum value of $q^{2}$ must occur on the boundary of any singularity-free region.

Since the Bernoulli equation is

$$
\frac{k}{k-1} \frac{p}{\rho}+\frac{q^{2}}{2}+g x_{3}=\text { constant }
$$

and the equation for isentropic change is

$$
p \rho^{-k}=p_{0} \rho_{0}^{-k}=\text { constant },
$$

minimum pressure, minimum density, and (from the equation of state for perfect gases) minimum temperature are always associated with the maximum value of

$$
q^{2}+2 g x_{3} \text {. }
$$

If now one takes the Laplacian of $q^{2}+2 g x_{3}$, one can show by a procedure strictly similar to the foregoing one that the maximum value of $q^{2}+2 g x_{3}$ must occur on the boundary of any singularity-free region. Thus for any such region of a steady subsonic flow, the minimum density, pressure, or temperature must occur on the boundary, provided the flow is irrotational and isentropic.

The boundary of a singularity-free region is, of course, not necessarily a solid boundary. However, in the case of an ambiently uniform flow past closed solid bodies placed in an infinite fluid, one may compare a streamline at infinity with another not entirely at infinity. Both streamlines can be considered to originate and terminate at the same potential planes (on each of which $\varphi$ is constant). Since there is variation of speed on the latter streamline, the maximum speed thereon must exceed the uniform fluid speed at infinity. This fact, plus the theorem for the speed just proved, shows that, for any ambiently uniform flow of the kind under discussion, the maximum speed must occur on one of the solid boundaries present in the flow if it is free from singularities-since the entire fluid space bounded by infinity and the solid boundaries can be taken to be the region under consideration.

A flow with uniform ambient velocity $U$ past a solid body is physically the same as one caused by that body moving with velocity $-U$ in an otherwise quiescent infinite fluid, i.e., the distributions of pressure and density relative to the body must be the same for both cases. Thus, if gravity is ignored, the minimum density, pressure or temperature in a subsonic flow caused by a body moving steadily in an otherwise quiescent fluid must occur on the boundary of that body if the flow is isentropic, irrotational, and free from singularities. 